\title{
Coding region structural heterogeneity and turnover of transcription start sites contribute to divergence in expression between duplicate genes Chungoo Park and Kateryna D Makova
}

\begin{abstract}
Address: Center for Comparative Genomics and Bioinformatics, Department of Biology, The Pennsylvania State University, University Park, PA 16802, USA.
\end{abstract}

Correspondence: Kateryna D Makova. Email: kdm16@psu.edu

Published: 28 January 2009

Genome Biology 2009, 10:RI0 (doi:I0.1 I86/gb-2009-10-I-r10)

The electronic version of this article is the complete one and can be found online at http://genomebiology.com/2009/I0/I/R 10
Received: II October 2008

Revised: 24 December 2008

Accepted: 28 January 2009

(C) 2009 Park and Makova; licensee BioMed Central Ltd.

This is an open access article distributed under the terms of the Creative Commons Attribution License (http://creativecommons.org/licenses/by/2.0), which permits unrestricted use, distribution, and reproduction in any medium, provided the original work is properly cited.

\begin{abstract}
Background: Gene expression divergence is one manifestation of functional differences between duplicate genes. Although rapid accumulation of expression divergence between duplicate gene copies has been observed, the driving mechanisms behind this phenomenon have not been explored in detail.

Results: We examine which factors influence expression divergence between human duplicate genes, utilizing the latest genome-wide data sets. We conclude that the turnover of transcription start sites between duplicate genes occurs rapidly after gene duplication and that gene pairs with shared transcription start sites have significantly higher expression similarity than those without shared transcription start sites. Moreover, we find that most (55\%) duplicate gene pairs do not retain the same coding sequence structure between the two duplicate copies and this also contributes to divergence in their expression. Furthermore, the proportion of aligned sequences in cis-regulatory regions between the two copies is positively correlated with expression similarity. Surprisingly, we find no effect of copy-specific transposable element insertions on the divergence of duplicate gene expression.
\end{abstract}

Conclusions: Our results suggest that turnover of transcription start sites, structural heterogeneity of coding sequences, and divergence of cis-regulatory regions between copies play a pivotal role in determining the expression divergence of duplicate genes.

\section{Background}

Because of the importance of gene duplication in evolution [15], it is crucial to know how duplicate genes diverge and which factors determine their destiny. Recently, genome-wide analyses of microarray data [6] have revealed patterns of expression divergence in duplicate genes, which are necessary for understanding the emergence of new functions after gene duplication. Numerous studies indicated that genes diverge rapidly in their expression after duplication [7-12]. Population genetic models proposed directional selection and relaxation of selective constraints as possible forces driving the evolution of expression in duplicate genes, although the relative frequency of these two scenarios in the evolution of paralogs is still being debated [4,5,13]. These population genetic 
models have been implemented under the assumption that two duplicated gene copies are structurally and functionally identical immediately after duplication. However, this assumption is sometimes violated. First, genes duplicated via retrotransposition lose regulatory sequences and include additional sequences at each side (for example, poly(A) tails at 3' terminus and short direct repeats at both termini), so that retrotransposed copies differ from the corresponding parental genes $[4,13,14]$. Second, tandem duplication by unequal crossing over might not include the entire coding sequence and/or regulatory elements specifying expression of a parental gene. Indeed, Katju and Lynch [15] demonstrated that more than half of newborn duplicate genes in Caenorhabditis elegans represent not complete, but rather partial or chimeric duplications. Such structural heterogeneity may play an important role in rapid expression divergence between human duplicate genes as well; however, it has not been considered in detail in previous studies.

Transposable elements (TEs) represent another factor that might account for the expression divergence of duplicate genes, since several studies provided evidence of TEs altering gene expression. Jordan and colleagues [16] showed that almost $25 \%$ of human promoter regions as well as many other cis-regulatory elements contain, or at least overlap with, TEderived sequences. This result was later confirmed by another study [17]. A specific example of the importance of TEs in the regulation of gene expression comes from the CYP19 gene, which encodes the aromatase enzyme, important for estrogen biosynthesis [18]. Because of the recent insertion of a long terminal repeat into the first exon of one of the isoforms of human CYP19, the gene gained expression in placenta, while its mouse ortholog has no long terminal repeat and is not expressed there [19].

Finally, alternative promoter usage by duplicate genes should be considered as a mechanism for rapid expression divergence. Recent comprehensive studies concluded that many known genes in the human genome are expressed from alternative promoters [20-23]. Similarly, approximately $22 \%$ of genes in the ENCODE regions have functional alternative promoters [24]. The alternative promoters provide a heterogeneity in tissue-specific expression patterns and levels, developmental activity, and translational efficiency [25-27]. As a result, the use of alternative promoters might be one of the major sources for achieving transcriptome diversity and one of the routes by which duplicate genes acquire divergence in their expression.

To investigate what drives expression divergence of human paralogs on a genome-wide scale, we addressed the following three questions in the present study: how frequently the turnover of transcription start sites (TSSs) occurs between duplicate genes; how often duplicate gene copies (their coding sequences) differ from each other structurally; and whether the density of copy-specific TEs within cis-regulatory regions influences expression divergence in duplicated genes. We utilized the gene expression profile available for 61 non-redundant and non-pathogenic human tissues [28], the largest comprehensive expression profile of human genes available to date, and assessed the contributions of TSS turnover, coding sequence structural heterogeneity, and TE integration to divergence in duplicate gene expression.

\section{Results \\ Identification of duplicate genes}

Utilizing two different methods, FASTA and TRIBE-MCL, we identified 6,536 and 7,027 non-redundant human duplicate gene pairs, respectively (see Materials and methods for details). These pairs represented 3,313 and 3,555 gene families, respectively. After filtering out duplicate gene pairs with synonymous rate $\left(K_{S}\right)>2$ and/or lacking a start codon, we obtained 2,790 and 2,750 duplicate gene pairs using the former and the latter methods, respectively. A total of 1,600 duplicate gene pairs overlapped between these two data sets (Additional data file 2). All subsequent analyses were carried out for duplicate genes identified with each of the two methods. Because the results were similar, we present the results only for duplicate genes identified with the FASTA method $(2,790$ gene pairs in group A), as this method is stricter for clustering proteins into families compared with the TRIBEMCL method [29,30].

From human U133A and GNF1H oligonucleotide arrays [28], we defined 14,505 genes that mapped to probes with a one-toone correspondence (see Materials and methods), thus minimizing cross-hybridization. Among these genes, we were able to detect 2,924 non-redundant duplicate gene pairs belonging to 1,792 multiple gene families. After filtering out duplicate gene pairs with $K_{S}>2$ and/or lacking a start codon, we obtained 1,015 duplicate gene pairs (group B, representing a subset of group A). In the remainder of the manuscript, we consider duplicate genes of group B when gene expression is investigated and duplicate genes of group A otherwise.

\section{Turnover of TSSs between duplicate genes}

Initially, we analyzed the divergence in the position of TSSs between copies in each duplicate gene pair. Using tag clusters, which were built by grouping overlapping tags (namely, 5 -end-sequences) with the same strand, from large-scale tag clustering of the cap analysis of gene expression (CAGE) [20] and the paired-end ditags (PETs) [31], putative TSSs of each gene were identified (see Materials and methods). From 2,790 duplicate gene pairs in group A, we excluded duplicate gene pairs that were duplicated by retrotransposition or for which at least one copy lacked a TSS(s) identified by either CAGE or PETs. As a result, 1,124 duplicate gene pairs were retained. To evaluate sharing of TSSs between duplicate genes, we compared the sequences of genomic regions surrounding putative TSSs (as identified by CAGE or PETs) between the two copies for each of these 1,124 duplicate gene 
pairs. We considered $110 \mathrm{bp}(-20 \mathrm{bp}$ to $+90 \mathrm{bp}$ ) surrounding each TSS (later called the 'TSS region'), because there was a clear peak in the average sequence similarity between TSSs of duplicate genes in this region (Additional data file 3) and because several studies indicated that a region of this size surrounding TSSs was well conserved between human and mouse orthologs [32,33]. Sequence similarity between all possible combinations of TSS regions from each duplicate gene pair was considered. If at least one pair of TSS regions had an identity greater than $60 \%$, it was defined as a TSS(s) shared between the two duplicate copies. As a result, 13.6\% (153 out of 1,124) of duplicate gene pairs had shared TSSs.

We observed that the relative frequency of gene pairs with shared TSSs decreases with increasing $K_{S}$, a proxy of time since duplication (Figure 1). The L-shaped distribution observed in Figure 1 implies a rapid turnover of TSSs after gene duplication. Already at $K_{S}=0.1$, corresponding to only about 33 million years ago since duplication [34], a mere $64 \%$ of duplicate genes share TSSs. Considering an instantaneous $K_{S}$ rate according to [35] did not alter our results (Additional data file 4).
Interestingly, the turnover of TSSs between human duplicate genes was much more rapid than between human-mouse orthologs. Indeed, for 1,610 human-mouse orthologs considered (see Materials and methods), the mean $K_{S}$ was 0.61 (with a $95 \%$ confidence interval of $0.60-0.63$ ), while the proportion of orthologs with shared TSSs was 0.71, several fold higher than the proportion of human duplicate genes with similar $K_{S}$ (Figure 1).

To estimate the relationship between TSS usage patterns (for example, shared TSSs versus non-shared TSSs) and gene duplication mechanisms, the duplicate genes were divided into three classes: retrotransposed duplicate genes, tandem, and nontandem duplications (see Materials and methods for details). The relative frequencies of gene pairs with shared TSSs in each class were calculated (thus, we analyzed 1,124 non-retransposed genes as above plus 220 retrotransposed genes). Duplicate gene copies in which one of the pair has one exon and the duplicate copy has multiple exons were called retrotransposed duplicate gene copies. We found that among paralogs with shared TSSs, the majority of pairs represented tandem duplicates (Additional data file 1).

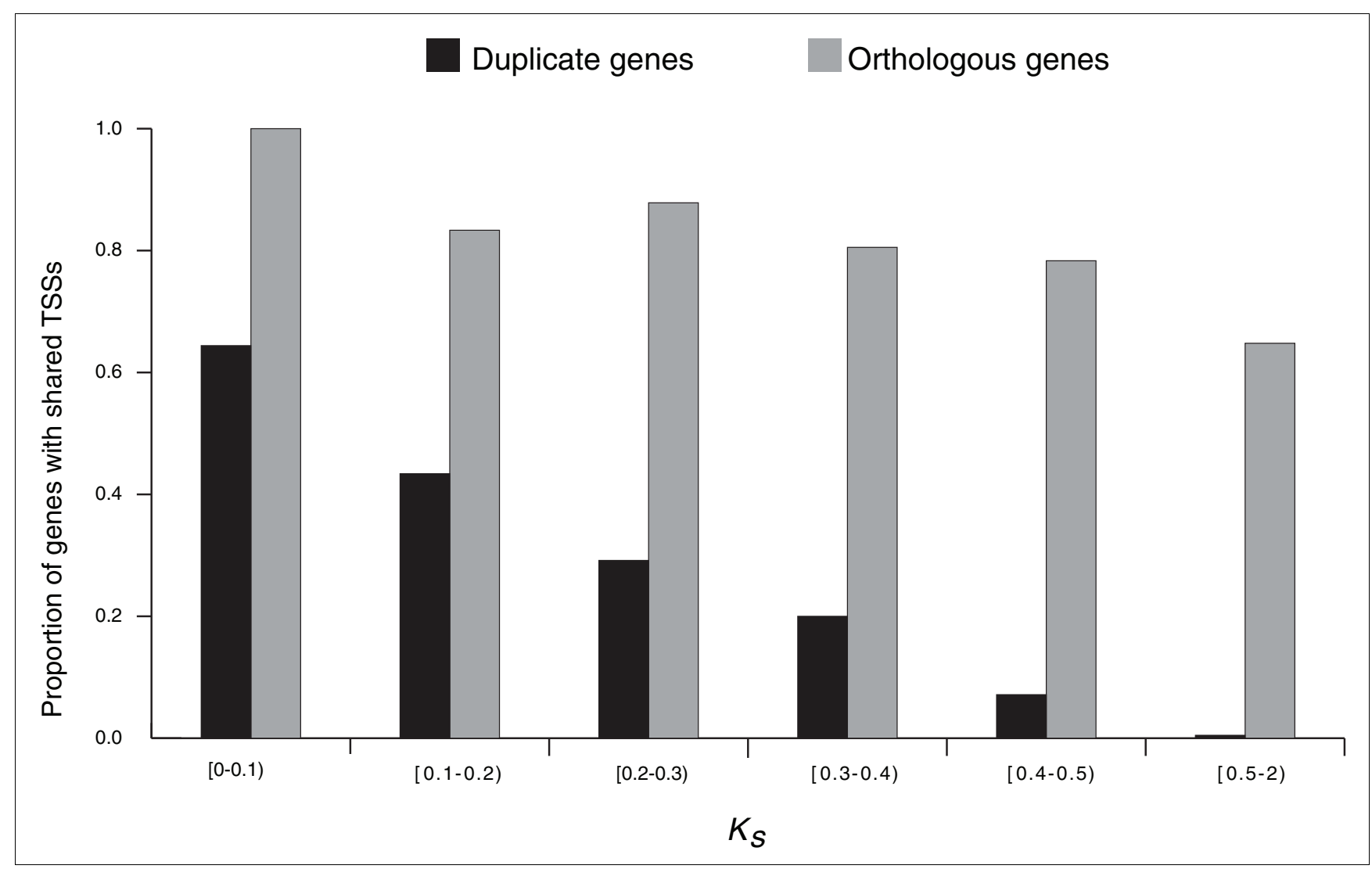

Figure I

The decline in the proportion of group A duplicate gene pairs with shared TSSs (shown in black) depending on the time since duplication (approximated by $K_{S}$ ). The proportion of human-mouse orthologous genes with conserved TSSs is shown for comparison (in gray); in this case variation in $K_{S}$ is due to regional variation in substitution rates. 
Interestingly, about 30\% (67 out of 220) of retrotransposed duplicate gene pairs retained the same TSSs (Additional data file 1). To evaluate whether the retrotransposed gene pairs with shared TSSs tend to undergo stronger purifying selection than those without shared TSSs, the median nonsynonymous-to-synonymous rate ratios $\left(K_{A} / K_{S}\right)$ were compared between these two groups of genes; however, no significant difference was detected (0.475 versus $0.499 ; P>0.1$, MannWhitney U test).

Next, to test whether the turnover of TSSs may contribute to the expression divergence in duplicate genes, the Pearson correlation coefficient of expression values $\left(R_{\text {expression }}\right.$; calculated for 61 non-redundant tissues) between the two copies in each pair was computed and compared among group B duplicate gene pairs with shared TSSs versus those without shared TSSs (a total of 581 group B pairs with available TSS data were included in the analysis). Duplicate genes with shared TSSs had significantly higher $R_{\text {expression }}$ values than those without shared TSSs (0.437 versus $0.080 ; P<0.01$, Mann-Whitney U test). It is conceivable that the significant difference in $R_{\text {expres- }}$ sion values is due to different synonymous rates in genes with shared TSSs versus those without shared TSSs. Indeed, we observed that all duplicate genes (belonging to group B) with shared TSSs had $K_{S}<0.4$, while more than $97 \%$ of gene pairs without shared TSSs had $K_{S} \geq 0.4$. However, if only genes with $K_{S}<0.4$ were considered, the gene pairs with shared TSSs still had higher (but not significantly so) $R_{\text {expression }}$ values than those without shared TSSs (0.437 versus $0.140 ; P>0.05$, Mann-Whitney U test).

The 60\% identity threshold among the TSS regions that was tentatively inferred from substitution rates between human and mouse ortholog core promoters [36] may be inadequate for estimating the sharing of TSSs among human paralogous genes. Thus, we reclassified the sharing of TSSs between copies of duplicate genes using several identity thresholds (40\%, $50 \%, 70 \%$, and $80 \%$ ). Although the numbers of duplicate genes with shared TSSs in each bin varied with the threshold, the frequency of gene pairs with shared TSSs decreased over divergent time independent of the threshold used (Additional data file 5), consistent with the pattern observed with the $60 \%$ identity threshold (Figure 1). Moreover, regardless of the identity threshold, the $R_{\text {expression }}$ values were significantly higher in duplicate genes with shared TSSs versus those without shared TSSs (data not shown).

\section{Structural heterogeneity in coding regions of human duplicate genes}

By reconstructing the full-length coding sequences via concatenating exons from multiple splicing variants for each gene separately, each pair of duplicate genes was classified into one of two structural categories: completely similar and incompletely similar. If the proportion of aligned sequences was greater than 0.9, duplicate gene pairs were categorized as completely similar and as incompletely similar otherwise. For some analyses, incompletely similar duplicate gene copies were classified in one of the three non-overlapping groups: $5^{\prime}$ similar, 3 ' similar, and neither 5 ' nor 3 ' similar. If alignments between the two copies started at the start codons of both copies, then such duplicates were classified as 5 ' similar. Alternatively, if the alignments ended at the stop codons of both copies, we classified the duplicate genes as 3' similar. The remaining duplicate gene pairs were labeled as neither $5^{\prime}$ nor 3' similar.

After excluding genes that lacked start/stop codons or consensus splice sites, 2,591 duplicate gene pairs were retained (from 2,790 pairs of group A; for group B, 889 duplicate gene pairs were retained). We found that $55 \%(1,429$ out of 2,591$)$ of duplicate gene pairs had incompletely similar structures. As expected from the divergence of the coding sequence over time, the proportion of duplicate gene pairs with completely similar structures decreased gradually with divergence between the two duplicate copies, approximated by $K_{S}$ (Figure 2). Considering an instantaneous $K_{S}$ rate according to [35] did not alter our results (Additional data file 6). Interestingly, even at the smallest duplicate gene divergence $\left(K_{S}<0.1\right)$, the proportion of genes with completely similar structures was only $80 \%$ (Figure 2). Although this finding might be affected by misannotations, our results suggest that some duplicate genes might have acquired structural differences during duplication.

To analyze whether the incompletely similar structures of duplicate genes can lead to expression divergence, we compared the relationship between $R_{\text {expression }}$ and $K_{S}$ for duplicate genes with completely versus incompletely similar structures. Before addressing this issue, retrotransposed duplicate genes (a total of 108 out of 889 genes retained in group B) were excluded because, as retrotransposition does not include a promoter, it can lead to expression divergence regardless of structural heterogeneity in coding sequence between duplicates. We found that: the correlation coefficient between $R_{e x-}$ pression and $K_{S}$ for duplicate gene pairs with completely similar structures was significantly lower than that for pairs with incompletely similar structures $(\mathrm{R}=-0.315$ versus $\mathrm{R}=-0.001$; Fisher's z test, $\mathrm{z}=-4.028, P<0.001$; Kolmogorov-Smirnov test for normality, $P<0.010$; Figure 3 and Table 1); and duplicate genes with completely similar structures had significantly higher y-intercepts of regression lines than duplicate genes with incompletely similar structures (0.407 versus $0.134 ; \mathrm{z}=2.672, P<0.01)$. These observations suggest that, immediately after duplication, the expression pattern is more similar for duplicate gene pairs retaining the same versus acquiring different coding sequence structures, and that divergence of gene expression is more dependent on evolutionary time for duplicate gene pairs with completely versus incompletely similar structures. To estimate the importance of sharing of 5 ' regions of coding sequences between duplicate gene copies, which can be an indirect indicator of common transcription regulation mechanisms, we separately consid- 


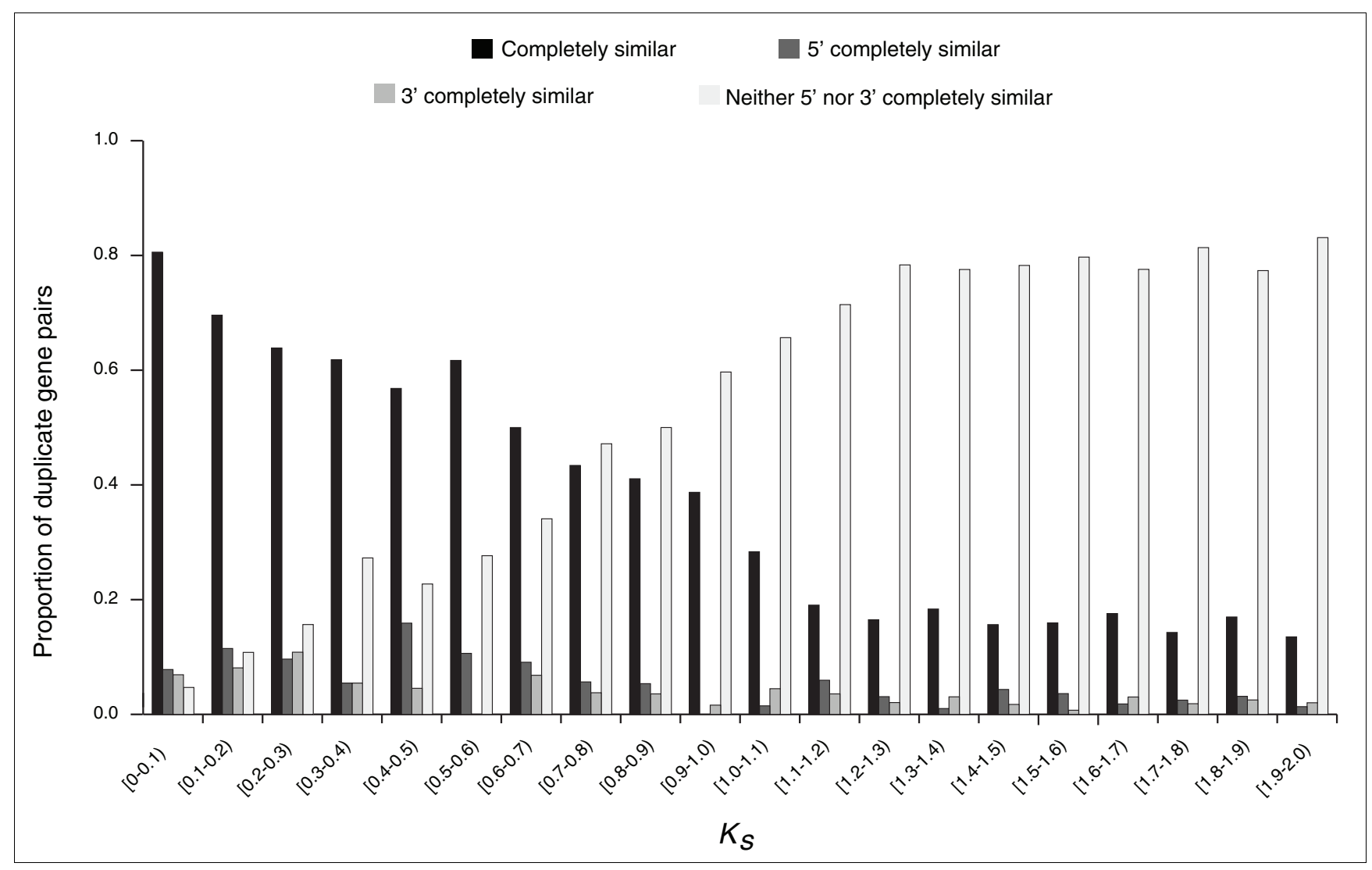

Figure 2

Proportion of group A duplicate gene pairs classified by coding sequence structural heterogeneity.

ered duplicate gene pairs completely similar at the 5 ' end only (a total of 24 gene pairs from group B that were otherwise genes with incompletely similar structures) and calculated the correlation coefficient between their $R_{\text {expression }}$ and $K_{S}$. The correlation was negative, but not significant (Table 1). When duplicate gene pairs having completely similar and 5 ' similar structures were considered together, the correlation coefficient between $R_{\text {expression }}$ and $K_{S}$ was somewhat lower than that for duplicate gene pairs with completely similar structures (Table 1), although the difference was not significant $(\mathrm{z}=-$ 0.093, $P>0.1$ ). We observed that there was no correlation between $R_{\text {expression }}$ and $K_{S}$ for duplicate genes with 3 ' similar structure and with neither 5 ' nor 3 ' similar structure (Table 1 ). These results suggest that maintenance of the entire coding region (and not just of its 5 ' or 3 ' portion) is important for determining gene expression profile after duplication.

To estimate differences in selective pressure among duplicate genes in different structural categories, their $K_{A} / K_{S}$ ratios were compared (Table 1 ). We observed that $K_{A} / K_{S}$ was significantly lower for duplicate genes with completely similar structures than for those with incompletely similar structures $(P<0.001$, Mann-Whitney U test; Table 1$)$, suggesting that the former genes are subject to stronger purifying selection than the latter genes.

\section{Divergence of cis-regulatory sequences between duplicate genes}

Next, we evaluated the relative contribution of cis-regulatory divergence to differences in expression between copies of duplicate genes in each pair. The $2-\mathrm{kb}$ (from $-1.5 \mathrm{~kb}$ to +0.5 $\mathrm{kb}$ ) genomic regions surrounding TSSs were used as putative cis-regulatory sequences and their divergence was estimated with REALIGNER [37]. For genes with multiple TSSs, a TSS supported by the highest number of CAGE/PET tags was selected. This analysis was limited to group B duplicate genes with completely similar structures (a total of 158 duplicate gene pairs). We found a significant positive correlation $(\mathrm{R}=$ $0.242, P<0.01)$ between the proportion of aligned sequences in the cis-regulatory region $\left(P_{c i s}\right)$ and $R_{\text {expression. }}$. This implies that the divergence of cis-regulatory regions leads to expression divergence in duplicate genes. After duplicate genes created by retrotransposition (a total of 23 gene pairs) were excluded, the correlation coefficient was even higher $(\mathrm{R}=$ 0.252, $P<$ 0.01). Through comparison between $K_{S}$ (which may serve as a neutral proxy, although see [38]) on the one hand and the proportion (corrected for multiple hits using 


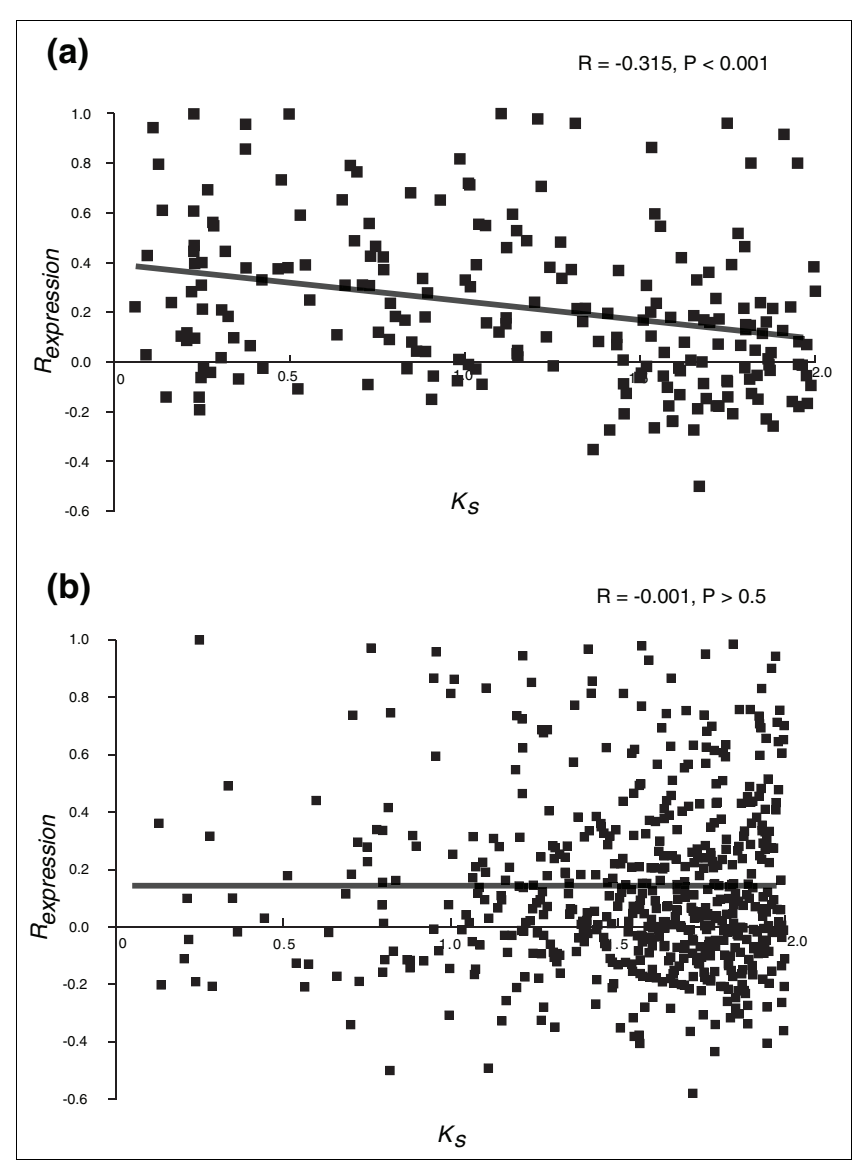

Figure 3

The relationship between $K_{S}$ and $R_{\text {expression }}$ for group $B$ duplicate genes with (a) completely similar structures and (b) incompletely similar structures.

HKY85 model) of aligned sequences in the cis-regulatory region on the other hand in each non-retrotransposed duplicate gene pair, we estimated whether the cis-regulatory regions evolved neutrally. We found that for 107 out of 135 duplicate gene pairs compared, $K_{S}$ was significantly higher $(P$ $<$ o.001, Wilcoxon signed-rank test) than the proportion of aligned sequences in the cis-regulatory region, suggesting that purifying selection acts at cis-regulatory regions.

To investigate whether copy-specific TEs influence divergence in duplicate gene expression, we identified such TEs (TEs that integrated in the cis-regulatory region of only one duplicate gene copy of a pair after duplication) in the same 2$\mathrm{kb}$ regions surrounding TSSs of the above 158 duplicate genes pairs (excluding 23 retrotransposed duplicate pairs; see Materials and methods). However, no significant correlation was found between the proportion of copy-specific TEs and either $P_{\text {cis }}$ for duplicate genes or $R_{\text {expression }}$ (data not shown). This suggests that the effect of copy-specific TEs on divergence in duplicate gene expression may be at best minor, although this issue requires additional studies.

\section{Interplay of multiple predictors in explaining divergence of paralogous gene expression}

Because several factors studied above might be interrelated, we conducted multiple regression analysis to estimate the relative contribution of each factor to explaining the total variability in $R_{\text {expression. }}$ A total of four continuous predictors $\left(K_{A}\right.$, $K_{S}$, the $K_{A} / K_{S}$ ratio, and divergence of cis-regulatory sequences (labeled 'Cis') and three categorical predictors (shared versus not shared TSSs (labeled 'TSS'); completely versus incompletely similar gene structure (labeled 'Structure'); and tandem versus non-tandem gene organization (labeled 'Tandem')) as well as all possible pairwise interaction terms were used to build a regression model. After pruning nonsignificant terms, the final multiple regression model explained approximately $10 \%$ of the variation in $R_{\text {expression }}$ and consisted of eight predictors (Table 2). Five of these predictors remained significant after applying Bonferroni correction for multiple tests (Table 2). These predictors included: Tandem, TSS, and interaction terms between Structure and Tandem, between TSS and Tandem, and between $K_{A} / K_{S}$ ratio and Cis (Table 2). Our computation of the relative contribution of the variability explained (RCVE) for significant predictors (see Materials and methods for details) indicated that each of them makes a sizeable input into the model.

Table I

The relationship between $K_{S}$ and $R_{\text {expression }}$ in each structural category using group B duplicate gene pairs

\begin{tabular}{|c|c|c|c|c|c|}
\hline Structural categories & Number of gene pairs & $K_{A} / K_{S}^{*}$ & $K_{s}^{*}$ & $R_{\text {expression }}{ }^{*}$ & $\begin{array}{c}\text { Pearson correlation coefficient of } K_{\mathrm{s}} \\
\text { versus } R_{\text {expression }}(P \text {-value })\end{array}$ \\
\hline Completely similar & 214 & $0.296(0.237)$ & $1.153(1.225)$ & $0.213(0.162)$ & $-0.315(<0.001)$ \\
\hline 5 ' similar & 24 & $0.391(0.311)$ & $1.292(1.501)$ & $0.053(0.026)$ & -0.157 (NS) \\
\hline 3' similar & 23 & $0.302(0.31 \mathrm{I})$ & $1.365(1.610)$ & $0.346(0.249)$ & 0.019 (NS) \\
\hline Neither 5 ' nor 3 ' similar & 520 & $0.551(0.456)$ & $1.565(1.658)$ & $0.126(0.063)$ & 0.017 (NS) \\
\hline $\begin{array}{l}\text { Incompletely similar } \\
\text { (the sum of the above three } \\
\text { categories) }\end{array}$ & 567 & $0.534(0.444)$ & $1.545(1.646)$ & $0.132(0.068)$ & -0.001 (NS) \\
\hline Completely and 5 similar & 238 & $0.307(0.246)$ & $1.167(1.263)$ & $0.197(0.15 \mathrm{I})$ & $-0.307(<0.001)$ \\
\hline
\end{tabular}

*Values are mean (median). NS, not significant. 
Table 2

\begin{tabular}{lcc}
\hline $\begin{array}{l}\text { Multiple regression models for expression divergence in duplicate } \\
\text { genes }\end{array}$ & $P$-value & RCVE* \\
\hline Predictors & $4.2 \times 10^{-2}(\mathrm{NS} \ddagger)$ & 0.075 \\
\hline Cis ${ }^{\dagger}$ & $9.9 \times 10^{-5}$ & 0.277 \\
TSS & $2.7 \times 10^{-6}$ & 0.405 \\
Tandem & $1.1 \times 10^{-2}(\mathrm{NS})$ & 0.118 \\
$K_{A} \times$ Cis & $2.7 \times 10^{-2}(\mathrm{NS})$ & 0.088 \\
$K_{S} \times$ Cis & $1.7 \times 10^{-3}$ & 0.180 \\
Structure $¥ \times$ Tandem & $1.1 \times 10^{-5}$ & 0.354 \\
TSS $\times$ Tandem & $3.1 \times 10^{-3}$ & 0.159 \\
$\omega^{\#} \times$ Cis & & 0.093 \\
$\mathrm{R}^{2}$ & &
\end{tabular}

*RCVE: relative contribution to the variability explained (see Materials and methods for more details). tCis: divergence of cis-regulatory sequences in $2 \mathrm{~kb}$ surrounding TSS (see Materials and methods for more details). $¥$ NS: not significant after Bonferroni correction for multiple tests. §TSS: shared versus not shared TSSs. ITandem: tandem versus nontandem organization of duplicate genes. $¥$ Structure: structural heterogeneity in coding sequences. ${ }^{\#} \omega: K_{A} / K_{S}$ ratio.

\section{Discussion}

Although it has been shown that duplicate genes diverge rapidly in their expression [10,39-41], little is known about which factors influence their expression divergence at the genomic level [42]. In this study, we investigated three such factors: structural heterogeneity of coding sequences, turnover of TSSs, and divergence of cis-regulatory regions (including insertions of copy-specific TEs).

Our results indicate that structural differences in coding sequences are common among human duplicate genes. We observed a high proportion of duplicate genes with structural differences even among young duplicates $\left(K_{S}<0.1\right)$, which is consistent with the findings for $C$. elegans duplicate genes [15]. Thus, genes might already be structurally different at the point of duplication. In general, duplication by unequal crossing over might not contain the entire coding sequence of a parental gene, and indeed, for the majority of individual young duplicate gene pairs with incompletely similar structures in our data set (for approximately 90\% of duplicate pairs of group A), both copies reside on the same chromosome. Over time, duplicate genes accumulate mutations leading to amino acid changes, premature stop codons, and atypical splicing $[4,14,43]$. These mutations might lead to decreasing numbers of duplicate genes retaining their ancestral structure and lead to more rapid divergence in expression and function.

Alteration of TSSs between duplicate gene copies is likely to have a direct impact on expression divergence. Using sequence similarity analysis, we examined whether duplicate genes share their TSSs. A large number of duplicate genes with distinct TSSs between the two copies were observed and these duplicate gene copies usually had different expression patterns. Although we did not directly estimate the fitness effects of turnover of TSSs on retention of duplicate genes, alteration of TSSs provides a means for the realization of several models of gene duplication evolution (for example, subfunctionalization and neofunctionalization [44,45]).

Additionally, we observed that cis-regulatory regions of duplicate genes diverge with time since duplication. This is consistent with several previous reports [46-48]. We investigated a potential impact of the density of copy-specific TEs on the divergence of duplicate gene expression and, surprisingly, found no major effect. This result corroborates recent findings regarding orthologous mammalian promoters; in human core promoters, the density of most observed repeat classes was significantly below the genomic average, suggesting that insertion of TEs in cis-regulatory regions is prevented by purifying selection [36].

Using multiple regression analysis, we observed that shared versus not shared TSS ('TSS'), completely versus incompletely similar structure ('Structure'), divergence of cis-regulatory sequences ('Cis'), the $K_{A} / K_{S}$ ratio, and tandem versus non-tandem duplicate gene organization played an important role in determining divergence in duplicate gene expression. It is worth noting that all three novel predictors introduced in this manuscript (TSS, Structure, and Cis) significantly influence divergence in duplicate gene expression alone and/or through interaction with other predictors. Interestingly, $K_{S}$, a proxy of evolutionary time, was not a significant predictor in our model. However, as noted above, evolutionary time influences alterations in other predictors and, therefore, the influence of $K_{S}$ on $R_{\text {expression }}$ might be observed through significance of predictors dependent on $K_{S}$. While interaction terms are not straightforward to interpret, the finding that several of them significantly contributed to the model suggests that considering multiple correlated factors might be essential for understanding patterns of duplicate gene expression divergence.

In this study, expression pattern was used as an indicator of evolution of biological functions after gene duplication. Several studies have suggested that gene expression density and breadth (for example, in housekeeping versus tissue-specific genes) has significantly influenced the evolution of proteins [49-52]. In addition to gene expression, which is likely a strong predictor [53,54], several additional factors have been implicated in protein evolution. Such factors include gene dispensability [55,56], protein stability and interaction network $[57,58]$ as well as codon usage $[54,59]$. Although these variables individually explain only a small fraction of variation in the rate of protein evolution, studying them might provide important insights into divergence between duplicate genes.

Most gene evolution models have assumed that two duplicate gene copies are expressed equally immediately after duplica- 
tion. However, similarly to coding sequences, promoter regions might also be incompletely duplicated between copies; this possibility needs to be evaluated in future studies. Frequently, because of the complex evolutionary dynamics of promoter sequences $[47,60,61]$, it is difficult to distinguish incomplete promoter duplication from rapid promoter evolution after duplication.

Reconstruction of ancestral gene expression state can be performed using a parsimony-based procedure in multi-gene families [62], instead of using the pairwise analysis employed here. However, rigorous filtering for potential cross-hybridization of transcripts of genes from the same multi-gene family in our study makes such ancestral reconstruction difficult. Thus, additional studies using different types of expression data may allow us to decompose the expression divergence of genes in multi-gene families and thus provide us with additional methodological insights for understanding gene expression divergence.

In the present study, as expected, we observed a significant negative correlation between the synonymous rate and Pearson correlation coefficient of expression values between duplicate gene copies; however, the resulting correlation was weaker than in our previous study [10]. There might be several potential reasons explaining this difference (for example, different $K_{S}$ thresholds used in the two studies and a greater number of tissues used in the present study). However, the major advance of the present study compared with the previous one [10] is a more rigorous filtering for potential crosshybridization of transcripts of two duplicate gene copies to the same probe, and thus we consider the present results more robust.

\section{Conclusion}

The present study represents the first report of the effects of structural differences in coding region and of unique TSSs on the divergence of duplicate gene expression. Our observations of frequent turnover of TSSs between duplicate genes and a high proportion of young duplicate genes with incompletely similar structures contradict the assumptions of classic gene duplication models, according to which duplicate genes are considered to be equal both structurally and functionally at the point of duplication $[4,13,14]$. Although potential incomplete duplication of promoters will be the subject of future studies, our investigation of factors contributing to expression divergence of duplicate genes provides important information for understanding human transcriptome heterogeneity, complexity, and evolution.

\section{Materials and methods Identification of duplicate gene pairs}

To cluster genes into families, we downloaded 48,218 protein sequences of consensus coding sequences, known and novel genes from Ensembl (release 38 of NCBI build 36) and independently used the FASTA [63] and TRIBE-MCL [64] methods to define duplicate gene families. Briefly, for the FASTA method, each protein sequence was used as a query to search against all other protein sequences using FASTA [65] with E $<$ 10. Two protein sequences formed a link if: the aligned region was $>80 \%$ of the longer protein; and the identity between two proteins was $\geq 30 \%$ for alignments longer than 150 amino acids or $\geq\left(0.01 \mathrm{n}+4.8 \mathrm{~L}^{-0.32}[1+\exp (-\mathrm{L} / 1000)]\right)$ otherwise, where $L$ is the alignable length between two proteins and $n=6$. The formula above was derived from empirical data, which suggested that a higher sequence identity was required for shorter proteins [66]. These gene pairs were grouped into gene families according to the single linkage clustering algorithm. For gene families derived by TRIBEMCL, we downloaded the gene annotations through BioMart in the Ensembl database, and considered gene families with at least two members.

To identify independent pairs of duplicate genes within each gene family, we sorted gene pairs in ascending order of $K_{S}$ and selected the pair with the lowest $K_{S}$. After excluding genes that had been picked, we chose the next gene pair with the lowest $K_{S}$. These steps were repeated for each gene family. All genes encoding proteins were realigned using CLUSTALW [67], and the ynoo module [68] of PAML [69] was used to calculate $K_{S}$. We counted duplicate gene pairs in intervals of size $K_{S}=0.01$ to derive the instantaneous rate of $K_{S}$ according to [35].

Duplicate gene copies in which one of the pair has one exon and the duplicate copy has multiple exons were called retrotransposed duplicate gene copies. In addition, duplicate gene pairs were classified as tandem duplicates if there were no genes separating them.

\section{Expression data analysis}

Expression data for 61 non-redundant and nonpathogenic human tissues in U133A and GNF1H Affymetrix arrays were obtained from [28]. To validate mapping between probe sets and genes, we aligned the transcripts of consensus coding sequences, known genes, and novel genes downloaded from Ensembl (release 38 of NCBI build 36) with the exemplar and consensus sequences for each array using BLAST [70] with E $<10^{-20}$. According to the criteria described in $[71,72]$, the acceptable alignments were selected if: the identity was $100 \%$ and the length was greater than $49 \mathrm{bp}$; or the identity was higher than $94 \%$ and the length was at least either 99 bp or $90 \%$ of the length of the query. We considered three scenarios for mapping relationships: a single probe set hitting one gene (9,508 probe sets); multiple probe sets hitting one gene (13,186 probe sets and 4,997 genes); and a single probe set hitting multiple genes (4,493 probe sets and 6,764 genes). All genes following the first two scenarios were utilized in the present study. For each gene following the second scenario, the probe set with the highest expression value (defined by 
average difference) was selected. All genes following the third scenario were removed from the analysis due to potential cross-hybridization. Following [28], genes with average difference $>\mathbf{2 0 0}$ in a particular tissue were considered to be expressed in this tissue.

\section{Identification of putative TSSs}

The putative TSSs were identified using the method described in the ENCODE pilot project [73]. Briefly, we utilized tag clusters from two sets of 5 -end-tag-capture technologies: CAGE [20] and PETs [31]. If two tag clusters were located on the same strand and within $60 \mathrm{bp}$ (which was derived from analyzing the distribution of distances between tag clusters in [73]) of each other, they were considered as one tag cluster. To map tag clusters to genes, the following two criteria were considered. First, the strand of a tag cluster was required to be identical to the strand of a gene. Second, a tag cluster was required to be located in the 5 ' upstream region from the most upstream start codon of a gene. Because we constructed artificial coding regions of genes by including all their exons, our analysis is not affected by alternative start codons. To confirm the reliability of the tag data, RefSeq [74], H-Invitational [75] and human ESTs [76] RNA data from the UCSC Genome Browser [77] were utilized. We excluded tag clusters with a single tag as well as those whose coordinates did not overlap with the genomic coordinates of the 5 ' end of cDNAs or ESTs. To define a representative tag site (to be used as a putative TSS) for each tag cluster, we selected the tag site that was supported by the highest number of 5 ' start sites. Otherwise, if several sites in a tag cluster had the same number of 5 ' start sites, the central coordinate of this tag cluster was defined as the representative tag site.

\section{Analysis of turnover of TSSs between human-mouse orthologous gene pairs}

To evaluate conservation of TSSs between human-mouse orthologous genes, we obtained two distinct classes of orthologous genes from [23]. Briefly, 'conserved promoter regions' means that upstream sequences of TSSs between human and mouse orthologous genes were aligned; otherwise, 'non-conserved promoter regions' means there were no significant alignments. We excluded orthologous genes that were classified into both classes because alternatively spliced variants of each gene had different conservation patterns of promoter regions. As a result, 1,610 orthologous gene pairs that were classified into just one class in a mutually exclusive manner were retained. We downloaded human and mouse protein sequences from Ensembl (release 38 of NCBI build 36). All genes were aligned using CLUSTALW [67], and the ynoo module [68] of PAML [69] was used to calculate $K_{S}$ between orthologous genes.

\section{Classification of the type of gene duplication into structural categories}

Structural categorization of duplicate genes was performed using reconstructed full-length coding sequences. We down- loaded annotated human genome data from Ensembl (release 38 of NCBI build 36). Alternatively spliced variants lacking start or stop codons or lacking canonical exon boundaries $\left(5^{\prime}-\right.$ GT...AG-3', 5'-GC...AG-3', or 5'-AT...AC-3') were excluded. For each gene with several alternatively spliced variants, all exons were aligned against each other, and, if some exons overlapped, they were merged in a single exon. Next, exons were sorted by their genomic coordinates and were reassembled to form reconstructed full-length coding sequences.

The reconstructed full-length coding sequences were aligned using AVID [78] with default parameters. Each pair of duplicate genes was classified into one of the four structural categories: completely similar, 5' similar, 3 ' similar, and neither 5 ' nor 3 ' similar. If the proportion of aligned sequences was greater than 0.9, duplicate gene pairs were categorized as completely similar. The other duplicate gene pairs were exclusively classified in just one category of 5 ' similar, 3 ' similar, or neither 5 ' nor 3 ' similar. If alignments between the two copies started at the start codons of both copies, then such duplicates were classified as 5 ' similar. Alternatively, if the alignments ended at the stop codons of both copies, we classified the duplicate genes into 3' similar. Finally, the remaining duplicate gene pairs were labeled as neither 5' nor 3' similar.

\section{Cis-regulatory regions analysis}

To detect homologous sequences in cis-regulatory regions, we used a modified version of REALIGNER [37]. Using BL2SEQ (part of the Blast suite [70]) with mismatch penalty equal to 2 and word size equal to 7 , we constructed alignments of $2-\mathrm{kb}$ $(-1.5 \mathrm{~kb}$ to $+0.5 \mathrm{~kb})$ genomic regions surrounding putative TSSs between copies in each duplicate gene pair. We selected alignments satisfying three criteria: hit length $>7 \mathrm{bp}$; identity $>70 \%$; and identical hit strand. If two local alignments overlapped, an alignment with the higher bit score was retained. If the bit scores of the two overlapping alignments were identical, a longer alignment or the one closest to TSS was retained. If the two local alignments were not syntenic (the order of blocks in each alignment was inconsistent), an alignment with the lower bit score was removed. Finally, all local alignments ordered by their genomic coordinates were used as a conserved cis-regulatory region for a duplicate gene pair.

TEs within cis-regulatory regions were classified into two sets: with the insertion occurring in the ancestral sequence before duplication of a genomic region; with the insertion in only one duplicate copy after the duplication event. We used the Repeatmasker [79] tables at the UCSC Genome Browser [77] to map the coordinates of TEs into cis-regulatory regions.

\section{Multiple regression analysis}

Linear multiple regression analysis was performed in the $\mathrm{R}$ statistical package. The original model included all seven predictors and their interaction terms, but was pruned to include only significant predictors (and significant interaction 
terms). RCVE $[80,81]$ was utilized to assess the contribution of each predictor to explaining the total variability:

$$
R C V E=\frac{R_{\text {full }}^{2}-R_{\text {reduced }}^{2}}{R_{\text {full }}^{2}}
$$

where $R_{\text {full }}^{2}$ and $R_{\text {reduced }}^{2}$ are the $R^{2}$ for the full model and the model except for the predictor of interest, respectively. In addition, variance inflation factors [82] were calculated for each predictor to diagnose multicollinearity. All predictors and their interaction terms included in the final model had variance inflation factors below 2 (data not shown), suggesting that multicollinearity was not adversely affecting the model.

\section{Abbreviations}

CAGE: cap analysis of gene expression; $K_{A}$ : nonsynonymous divergence; $K_{S}$ : synonymous rate; PET: paired-end ditag; RCVE: relative contribution to variability explained; TE: transposable element; TSS: transcription start site.

\section{Authors' contributions}

$\mathrm{CP}$ and KDM designed the experiments and wrote the manuscript. CP performed data analyses.

\section{Additional data files}

The following additional data are available with the online version of this paper. Additional data file 1 is a table listing the classification of duplicate gene pairs based on the absence or presence of shared TSSs and different duplication mechanisms. Additional data file 2 is a Venn diagram depicting the number of duplicate gene pairs that were identified by the FASTA and TRIBE-MCL methods. Additional data file 3 shows average sequence identity between TSS regions of duplicate genes. Additional data file 4 shows number of duplicate gene pairs with shared TSSs (A) and without shared TSSs (B) plotted against the instantaneous rate of $K_{S}$. Additional data file 5 shows proportions of group A duplicate gene pairs with shared TSSs depending on different identity thresholds. Additional data file 6 shows number of duplicate gene pairs in different structure categories plotted against the instantaneous rate of $K_{S}$.

\section{Acknowledgements}

We thank Ross Hardison, Webb Miller, Francesca Chiaromonte, Laura Carrel, and Claude dePamphilis for valuable discussions. We are grateful to Melissa Wilson for comments on the manuscript. This work was supported by start-up funds from Penn State (to KDM).

\section{References}

I. Ohno S: Evolution by Gene Duplication New York: Springer Verlag; 1970.

2. Taylor JS, Raes J: Duplication and divergence: the evolution of new genes and old ideas. Annu Rev Genet 2004, 38:6I5-643.

3. Wagner A: Selection and gene duplication: a view from the genome. Genome Biol 2002, 3:reviews I0I2.

4. Zhang J: Evolution by gene duplication: an update. Trends Ecol Evol 2003, 18:292-298.

5. Lynch M, Conery JS: The evolutionary fate and consequences of duplicate genes. Science 2000, 290: II5I-II55.

6. Shiu SH, Borevitz JO: The next generation of microarray research: applications in evolutionary and ecological genomics. Heredity 2008, 100:141-149.

7. Conant GC, Wagner A: Asymmetric sequence divergence of duplicate genes. Genome Res 2003, 13:2052-2058.

8. Gu X, Zhang Z, Huang W: Rapid evolution of expression and regulatory divergences after yeast gene duplication. Proc Natl Acad Sci USA 2005, 102:707-7I2.

9. Gu Z, Nicolae D, Lu HH, Li WH: Rapid divergence in expression between duplicate genes inferred from microarray data. Trends Genet 2002, 18:609-6I3.

10. Makova KD, Li WH: Divergence in the spatial pattern of gene expression between human duplicate genes. Genome Res 2003, 13:1638-1645.

II. Wagner A: Decoupled evolution of coding region and mRNA expression patterns after gene duplication: implications for the neutralist-selectionist debate. Proc Natl Acad Sci USA 2000, 97:6579-6584.

12. Zhang P, Gu Z, Li WH: Different evolutionary patterns between young duplicate genes in the human genome. Genome Biol 2003, 4:R56.

13. Lynch M, Katju V: The altered evolutionary trajectories of gene duplicates. Trends Genet 2004, 20:544-549.

14. Hurles M: Gene duplication: the genomic trade in spare parts. PLoS Biol 2004, 2:E206.

15. Katju $V$, Lynch M: The structure and early evolution of recently arisen gene duplicates in the Caenorhabditis elegans genome. Genetics 2003, 165: 1793-1803.

16. Jordan IK, Rogozin IB, Glazko GV, Koonin EV: Origin of a substantial fraction of human regulatory sequences from transposable elements. Trends Genet 2003, 19:68-72.

17. Thornburg BG, Gotea V, Makalowski W: Transposable elements as a significant source of transcription regulating signals. Gene 2006, 365:104-110.

18. Kamat A, Hinshelwood MM, Murry BA, Mendelson CR: Mechanisms in tissue-specific regulation of estrogen biosynthesis in humans. Trends Endocrinol Metab 2002, 13:122-128.

19. Lagemaat LN van de, Landry JR, Mager DL, Medstrand P: Transposable elements in mammals promote regulatory variation and diversification of genes with specialized functions. Trends Genet 2003, 19:530-536.

20. Carninci P, Sandelin A, Lenhard B, Katayama S, Shimokawa K, Ponjavic J, Semple CA, Taylor MS, Engstrom PG, Frith MC, Forrest AR, Alkema WB, Tan SL, Plessy C, Kodzius R, Ravasi T, Kasukawa T, Fukuda S, Kanamori-Katayama M, Kitazume Y, Kawaji H, Kai C, Nakamura M, Konno H, Nakano K, Mottagui-Tabar S, Arner P, Chesi A, Gustincich $S$, Persichetti $F$, et al:: Genome-wide analysis of mammalian promoter architecture and evolution. Nat Genet 2006, 38:626-635.

21. Kim TH, Barrera LO, Zheng M, Qu C, Singer MA, Richmond TA, Wu $Y$, Green RD, Ren B: A high-resolution map of active promoters in the human genome. Nature 2005, 436:876-880.

22. Kimura K, Wakamatsu A, Suzuki Y, Ota T, Nishikawa T, Yamashita R, Yamamoto J, Sekine M, Tsuritani K, Wakaguri H, Ishii S, Sugiyama T, Saito K, Isono Y, Irie R, Kushida N, Yoneyama T, Otsuka R, Kanda K, Yokoi T, Kondo H, Wagatsuma M, Murakawa K, Ishida S, Ishibashi T, Takahashi-Fujii A, Tanase T, Nagai K, Kikuchi H, Nakai K, et al:: Diversification of transcriptional modulation: large-scale identification and characterization of putative alternative promoters of human genes. Genome Res 2006, 16:55-65.

23. Tsuritani K, Irie T, Yamashita R, Sakakibara $Y$, Wakaguri $H$, Kanai $A$, Mizushima-Sugano J, Sugano S, Nakai K, Suzuki Y: Distinct class of putative "non-conserved" promoters in humans: comparative studies of alternative promoters of human and mouse genes. Genome Res 2007, 17:1005-1014.

24. Cooper SJ, Trinklein ND, Anton ED, Nguyen L, Myers RM: Compre- 
hensive analysis of transcriptional promoter structure and function in $1 \%$ of the human genome. Genome Res 2006, 16:1-10.

25. Landry JR, Mager DL, Wilhelm BT: Complex controls: the role of alternative promoters in mammalian genomes. Trends Genet 2003, 19:640-648.

26. Strausberg RL, Levy S: Promoting transcriptome diversity. Genome Res 2007, I7:965-968.

27. Trinklein ND, Aldred SJ, Saldanha AJ, Myers RM: Identification and functional analysis of human transcriptional promoters. Genome Res 2003, I3:308-312.

28. Su Al, Wiltshire T, Batalov S, Lapp H, Ching KA, Block D, Zhang J, Soden R, Hayakawa M, Kreiman G, Cooke MP, Walker JR, Hogenesch JB: A gene atlas of the mouse and human protein-encoding transcriptomes. Proc Natl Acad Sci USA 2004, I I I:6062-6067.

29. Horan K, Lauricha J, Bailey-Serres J, Raikhel N, Girke T: Genome cluster database. A sequence family analysis platform for Arabidopsis and rice. Plant Physiol 2005, 138:47-54.

30. Yang J, Lusk R, Li WH: Organismal complexity, protein complexity, and gene duplicability. Proc Natl Acad Sci USA 2003, 100:1566I-15665.

31. Ng P, Wei CL, Sung WK, Chiu KP, Lipovich L, Ang CC, Gupta S, Shahab A, Ridwan A, Wong CH, Liu ET, Ruan Y: Gene identification signature (GIS) analysis for transcriptome characterization and genome annotation. Nat Methods 2005, 2:105-III.

32. Suzuki $Y$, Yamashita R, Shirota M, Sakakibara Y, Chiba J, MizushimaSugano J, Nakai K, Sugano S: Sequence comparison of human and mouse genes reveals a homologous block structure in the promoter regions. Genome Res 2004, I4:17। I-1718.

33. Jin VX, Singer GA, Agosto-Perez FJ, Liyanarachchi S, Davuluri RV: Genome-wide analysis of core promoter elements from conserved human and mouse orthologous pairs. $B M C$ bioinformatics 2006, 7:114.

34. Yi S, Ellsworth DL, Li WH: Slow molecular clocks in Old World monkeys, apes, and humans. Mol Biol Evol 2002, 19:2191-2198.

35. Hughes T, Liberles DA: The pattern of evolution of smallerscale gene duplicates in mammalian genomes is more consistent with neo- than subfunctionalisation. J Mol Evol 2007, 65:574-588.

36. Taylor MS, Kai C, Kawai J, Carninci P, Hayashizaki Y, Semple CA: Heterotachy in mammalian promoter evolution. PLoS Genet 2006, 2:e30.

37. Iwama $H$, Gojobori $T$ : Highly conserved upstream sequences for transcription factor genes and implications for the regulatory network. Proc Natl Acad Sci USA 2004, 10 I: 17 I56-17161.

38. Chamary JV, Parmley JL, Hurst LD: Hearing silence: non-neutral evolution at synonymous sites in mammals. Nat Rev Genet 2006, 7:98-108.

39. Li WH, Yang J, Gu X: Expression divergence between duplicate genes. Trends Genet 2005, 21:602-607.

40. Scannell DR, Wolfe KH: A burst of protein sequence evolution and a prolonged period of asymmetric evolution follow gene duplication in yeast. Genome Res 2008, 18:137-147.

41. Semon M, Wolfe KH: Preferential subfunctionalization of slowevolving genes after allopolyploidization in Xenopus laevis . Proc Natl Acad Sci USA 2008, 105:8333-8338.

42. Ha M, Li WH, Chen ZJ: External factors accelerate expression divergence between duplicate genes. Trends Genet 2007, 23:162-166.

43. Prince VE, Pickett FB: Splitting pairs: the diverging fates of duplicated genes. Nat Rev Genet 2002, 3:827-837.

44. Lynch M, Force $A$ : The probability of duplicate gene preservation by subfunctionalization. Genetics 2000, 154:459-473.

45. Shiu SH, Byrnes JK, Pan R, Zhang P, Li WH: Role of positive selection in the retention of duplicate genes in mammalian genomes. Proc Natl Acad Sci USA 2006, 103:2232-2236.

46. Papp $\mathrm{B}, \mathrm{Pal} \mathrm{C}$, Hurst LD: Evolution of cis-regulatory elements in duplicated genes of yeast. Trends Genet 2003, 19:417-422.

47. Castillo-Davis $\mathrm{Cl}$, Hartl DL, Achaz G: cis-Regulatory and protein evolution in orthologous and duplicate genes. Genome Res 2004, I 4: 1530-1536.

48. Leach LJ, Zhang Z, Lu C, Kearsey MJ, Luo Z: The role of cis-regulatory motifs and genetical control of expression in the divergence of yeast duplicate genes. Mol Biol Evol 2007, 24:2556-2565.

49. Zhang L, Li WH: Mammalian housekeeping genes evolve more slowly than tissue-specific genes. Mol Biol Evol 2004, 2 I:236-239.

50. Subramanian S, Kumar S: Gene expression intensity shapes evolutionary rates of the proteins encoded by the vertebrate genome. Genetics 2004, 168:373-38I.

5I. Pal C, Papp B, Hurst LD: Highly expressed genes in yeast evolve slowly. Genetics 200I, 158:927-931.

52. Duret L, Mouchiroud D: Determinants of substitution rates in mammalian genes: expression pattern affects selection intensity but not mutation rate. Mol Biol Evol 2000, 17:68-74.

53. Drummond DA, Raval A, Wilke CO: A single determinant dominates the rate of yeast protein evolution. Mol Biol Evol 2006, 23:327-337.

54. Rocha EP, Danchin A: An analysis of determinants of amino acids substitution rates in bacterial proteins. Mol Biol Evol 2004, $21: 108-116$.

55. Hirsh AE, Fraser HB: Protein dispensability and rate of evolution. Nature 200 I, 4I I: 1046-1049.

56. Zhang J, He X: Significant impact of protein dispensability on the instantaneous rate of protein evolution. Mol Biol Evol 2005, 22:1 I 47-III55.

57. Fraser HB, Hirsh AE, Steinmetz LM, Scharfe C, Feldman MW: Evolutionary rate in the protein interaction network. Science 2002, 296:750-752.

58. Drummond DA, Wilke CO: Mistranslation-induced protein misfolding as a dominant constraint on coding-sequence evolution. Cell 2008, 134:34|-352.

59. Wall DP, Hirsh AE, Fraser HB, Kumm J, Giaever G, Eisen MB, Feldman MW: Functional genomic analysis of the rates of protein evolution. Proc Natl Acad Sci USA 2005, 102:5483-5488.

60. Cusack BP, Wolfe KH: Not born equal: increased rate asymmetry in relocated and retrotransposed rodent gene duplicates. Mol Biol Evol 2007, 24:679-686.

61. Liang H, Lin YS, Li WH: Fast evolution of core promoters in primate genomes. Mol Biol Evol 2008, 25:1239-1244.

62. Rossnes R, Eidhammer I, Liberles DA: Phylogenetic reconstruction of ancestral character states for gene expression and mRNA splicing data. BMC Bioinformatics 2005, 6: I 27.

63. Gu Z, Cavalcanti A, Chen FC, Bouman P, Li WH: Extent of gene duplication in the genomes of Drosophila, nematode, and yeast. Mol Biol Evol 2002, 19:256-262.

64. Enright AJ, Van Dongen S, Ouzounis CA: An efficient algorithm for large-scale detection of protein families. Nucleic Acids Res 2002, 30: $1575-1584$

65. Pearson WR, Lipman DJ: Improved tools for biological sequence comparison. Proc Natl Acad Sci USA 1988, 85:2444-2448.

66. Rost B: Twilight zone of protein sequence alignments. Protein Eng 1999, I 2:85-94.

67. Thompson JD, Higgins DG, Gibson TJ: CLUSTAL W: improving the sensitivity of progressive multiple sequence alignment through sequence weighting, position-specific gap penalties and weight matrix choice. Nucleic Acids Res 1994, 22:4673-4680.

68. Yang Z, Nielsen R: Estimating synonymous and nonsynonymous substitution rates under realistic evolutionary models. Mol Biol Evol 2000, 17:32-43.

69. Yang Z: PAML: a program package for phylogenetic analysis by maximum likelihood. Comput Appl Biosci 1997, 13:555-556.

70. Altschul SF, Madden TL, Schaffer AA, Zhang J, Zhang Z, Miller W, Lipman DJ: Gapped BLAST and PSI-BLAST: a new generation of protein database search programs. Nucleic Acids Res 1997, 25:3389-3402.

7I. Chung WY, Albert R, Albert I, Nekrutenko A, Makova KD: Rapid and asymmetric divergence of duplicate genes in the human gene coexpression network. BMC Bioinformatics 2006, 7:46.

72. Huminiecki L, Lloyd AT, Wolfe KH: Congruence of tissue expression profiles from Gene Expression Atlas, SAGEmap and Tissuelnfo databases. BMC Genomics 2003, 4:3I.

73. Birney E, Stamatoyannopoulos JA, Dutta A, Guigo R, Gingeras TR, Margulies EH, Weng Z, Snyder M, Dermitzakis ET, Thurman RE, Kuehn MS, Taylor CM, Neph S, Koch CM, Asthana S, Malhotra A, Adzhubei I, Greenbaum JA, Andrews RM, Flicek P, Boyle PJ, Cao H, Carter NP, Clelland GK, Davis S, Day N, Dhami P, Dillon SC, Dorschner MO, Fiegler $\mathrm{H}$, et al: Identification and analysis of functional elements in $1 \%$ of the human genome by the ENCODE pilot project. Nature 2007, 447:799-816.

74. Pruitt KD, Tatusova T, Maglott DR: NCBI reference sequences (RefSeq): a curated non-redundant sequence database of genomes, transcripts and proteins. Nucleic Acids Res 2007, 35:D6I-65.

75. Imanishi T, Itoh T, Suzuki Y, O'Donovan C, Fukuchi S, Koyanagi KO, Barrero RA, Tamura T, Yamaguchi-Kabata Y, Tanino M, Yura K, Miyazaki S, Ikeo K, Homma K, Kasprzyk A, Nishikawa T, Hirakawa M, Thi- 
erry-Mieg J, Thierry-Mieg D, Ashurst J, Jia L, Nakao M, Thomas MA, Mulder N, Karavidopoulou Y, Jin L, Kim S, Yasuda T, Lenhard B, Eveno E, et al.: Integrative annotation of 2I,037 human genes validated by full-length cDNA clones. PLOS Biol 2004, 2:el 62

76. Benson DA, Karsch-Mizrachi I, Lipman DJ, Ostell J, Wheeler DL: GenBank: update. Nucleic Acids Res 2004, 32:D23-26.

77. Karolchik D, Kuhn RM, Baertsch R, Barber GP, Clawson H, Diekhans M, Giardine B, Harte RA, Hinrichs AS, Hsu F, Kober KM, Miller W, Pedersen JS, Pohl A, Raney BJ, Rhead B, Rosenbloom KR, Smith KE, Stanke M, Thakkapallayil A, Trumbower H, Wang T, Zweig AS, Haussler D, Kent W]: The UCSC Genome Browser Database: 2008 update. Nucleic Acids Res 2008, 36:D773-779.

78. Bray N, Dubchak I, Pachter L: AVID: A global alignment program. Genome Res 2003, 13:97-102.

79. Jurka J: Repbase update: a database and an electronic journal of repetitive elements. Trends Genet 2000, 16:418-420.

80. Kvikstad EM, Tyekucheva S, Chiaromonte F, Makova KD: A macaque's-eye view of human insertions and deletions: differences in mechanisms. PLoS Comput Biol 2007, 3:1772-1782.

8I. Kelkar YD, Tyekucheva S, Chiaromonte F, Makova KD: The genome-wide determinants of human and chimpanzee microsatellite evolution. Genome Res 2008, 18:30-38.

82. Kutner MH, Nachtsheim CJ, Neter J, Li W: Applied Linear Statistical Models New York: McGraw-Hill; 2005. 